\title{
Percutaneous direct current stimulation - a new electroceutical solution for severe neurological pain and soft tissue injuries
}

This article was published in the following Dove Press journal:

Medical Devices: Evidence and Research

\author{
Albrecht Molsberger ${ }^{1,2, *}$ \\ Colin D McCaig',* \\ 'Department of Orthopedics, \\ Ruhr-University Bochum, Bochum, \\ Germany; ${ }^{2}$ Clinic for Orthopedics and \\ Pain Treatment, Düsseldorf, Germany; \\ ${ }^{3}$ Institute of Medical Sciences, \\ University of Aberdeen, Aberdeen, \\ Scotland \\ *These authors contributed equally to \\ this work
}

\begin{abstract}
There is a high medical need to improve the effectiveness of the treatment of pain and traumatic soft tissue injuries. In this context, electrostimulating devices have been used with only sporadic success. There is also much evidence of endogenous electrical signals that play key roles in regulating the development and regeneration of many tissues. Transepithelial potential gradients are one source of the direct current (DC) electrical signals that stimulate and guide the migration of inflammatory cells, epithelial cells, fibroblasts and mesenchymal stem cells to achieve effective wound healing. Up to now, this electrophysiological knowledge has not been adequately translated into a clinical treatment. Here, we present a mobile, handheld electroceutical smart device based on a microcontroller, an analog front end and a battery, which generates DC electric fields (EFs), mimicking and modulating the patient's own physiological electrical signals. The electrical stimulation is applied to percutaneous metal probes, which are located close to the inflamed or injured tissue of the patient. The treatment can be used in an ambulatory or stationary environment. It shows unexpectedly, highly effective treatment for certain severe neurological pain conditions, as well as traumatic soft tissue injuries (muscle/ ligament ruptures, joint sprains). Without EF intervention, these conditions, respectively, are either virtually incurable or take several months to heal. We present three cases - severe chronic cluster headache, acute massive muscle rupture of the rectus femoris and an acute ankle sprain with a ruptured anterior talofibular ligament - to demonstrate clinical effectiveness and discuss the fundamental differences between mimicking DC simulation and conventional transcutaneous electric nerve stimulation (TENS) or TENS-like implanted devices as used for peripheral nerve cord, spinal cord or dorsal root stimulation.
\end{abstract}

Keywords: electroceutical device, tissue regeneration, pain, direct current stimulation, electric field

\section{Introduction}

A range of electrostimulating devices is used for pain treatment. For transcutaneous electric nerve stimulation (TENS), percutaneous stimulation (e.g., electroacupuncture) or surgically implantable devices such as peripheral nerve stimulators (PNS), spinal cord stimulators (SCS) or dorsal root ganglion (DRG) stimulators, the method is basically the same. Dynamic, pulsed bipolar electric stimuli (1-70 V, 1-90 mA, $1-1200 \mathrm{~Hz}$, pulse width of $0.2-250 \mathrm{~ms}$ ) are applied to override the electrical capacity of nerves to generate further action potentials, thus interrupting the afferent pathways in locomotive or neurological pain conditions. These electrical devices are widely established clinically, although their clinical efficacy is not unequivocally proven,
Correspondence: Albrecht Molsberger Clinic for Orthopedics and Pain

Treatment, Kasernenstraße Ib, 40213

Düsseldorf, Germany

Tel +49 2II 866880

Email albrechtmolsberger@mac.com 
and the treatment only aims at suppressing pain. ${ }^{1-3}$ There is no clinical claim or evidence that TENS or TENS-like treatments improve regeneration or healing. Another class of electrostimulating devices aims at thermal or nonthermal electrochemical ablation (percutaneous thermocoagulation or electrolysis) to reduce anatomical pressure from a nerve, to ablate nociception or to promote consecutive angiogenesis and tissue repair. ${ }^{4,5}$

However, electrophysiological phenomena are not restricted to dynamic action potentials. Both in the extracellular matrix and within cells, ions and proteins/peptides are charged, and ion channels and ion transporters in the cell membrane regulate the flow of these charged molecules. Each tissue produces ionic currents and consequently electric fields (EFs) with a defined orientation and intensity. These EFs interact with charged ions and peptides by electrostatic forces, electrophoresis and electro-osmosis. ${ }^{6}$ For example, muscle-tissue inflammation as observed in painful trigger points is associated with a locally increased concentration of proinflammatory cytokines and $\mathrm{H}^{+}$ions, resulting in acidosis and local changes in electrical properties that favor increased electrical conductivity of the inflamed tissue. ${ }^{7}$

Another example is the transepithelial potential (TEP) difference. In epithelial tissues, epithelial cells frequently generate a net influx of $\mathrm{Na}^{+}$ions toward the parenchyma. Thus, epithelial cells, which are linked to each other by tight junctions that maintain ionic differences between the inner and outer aspects of the epithelium, create multiple, parallel and interlinked microbatteries that generate the TEP difference. ${ }^{8}$ Epithelial wounds cause a local collapse of the electrical resistance and a short-circuiting of the TEP locally because of the destruction of the tight junction seals and the build-up of edema. Depending on the size and location of the wound, the species and the specific tissue injured, this would induce small local direct currents (DCs); in human beings, these electrical signals are of the order of $140 \mathrm{mV} /$ $\mathrm{mm}$ and the drive current is $\sim 20-50 \mu \mathrm{A} / \mathrm{cm} .^{2,8} \mathrm{~A}$ large body of evidence proves that these small wound-generated DC EFs are predominant directional cues for wound healing and tissue regeneration. ${ }^{9-11}$ DC EFs guide and stimulate the migration of inflammatory cells, epithelial cells and fibroblasts. ${ }^{12,13}$ Depending on the orientation and intensity of the EFs, fibroblasts migrate toward or away from the wound, leading to an opening or closing of the wound. ${ }^{13}$ Small DC EFs also stimulate and direct the growth of spinal neurons (Borgens), astrocytes (Baer), mesenchymal stem cells (Zhao), monocytes and macrophages (Hoare), thus steering the process of regeneration of different tissues in mammals. ${ }^{14-17}$
This recently gained electrophysiological knowledge has inspired new clinical applications to improve regeneration. Tissue engineering is making huge strides, and new electrical devices are being tested to improve wound healing. ${ }^{18}$ However, we believe the clinical impact could and should be much wider.

In this article, we present a patented electroceutical treatment device that for the first time translates the knowledge of small steady DC EFs into the clinical treatment of neurologic and locomotive disorders. In contrast to the electrical stimulator devices described earlier, this electrical stimulator delivers a unipolar, steady DC over 30 minutes duration, which mimics and increases physiological EFs to modulate local tissue inflammation and to trigger the regeneration of nerves, muscles, ligaments and tendons. Selected case histories are presented to exemplify the treatment procedure.

\section{Materials and methods}

In developing the device we faced four challenges. First, between electrical probes inserted into the subcutaneous tissue or placed on the skin, a time- and voltage-dependent electrical resistance is observed, resulting in an unstable current over the time of stimulation. Second, the active stimulation electrode most commonly should be the cathode, inducing the desired buffering of acidosis and creating an EF to attract cells by galvanotaxis, whereas the indifferent electrode should be the anode. Third, for the anode, we had to find a solution for the oxidative electrolysis that induces acidosis and oxidative interaction with the stainless steel alloy, transporting metal ions into the tissue. Finally, sensorimotor irritation could be observed when switching a DC current on and off in the human body.

It was decided to develop a mobile handheld smart device, based on a microcontroller, an analog front end and a battery (Figure 1). The device generates a constant DC with predefined and selectable values up to $540 \mu \mathrm{A}$ for a treatment time of 30 minutes. The current is delivered to the patient by an anodal skin pad electrode and a cathodal subcutaneous/intramuscular set of one to nine stainless steel needle electrodes. The current output is selected depending on the needle diameter, tissue, body region and type of disease.

To avoid sensory effects at the start and end of the treatment, a ramping in/out of the selected current was programmed. To counteract possible habituation, the amplitude of the DC current was modulated by a small $( \pm 10 \%)$ and slow $(0.1 \mathrm{~Hz})$ alternating current $(\mathrm{AC})$.

The given requirements are mapped by a voltage signal $u(t)$ depicted in Equation 1. The described medical device is 


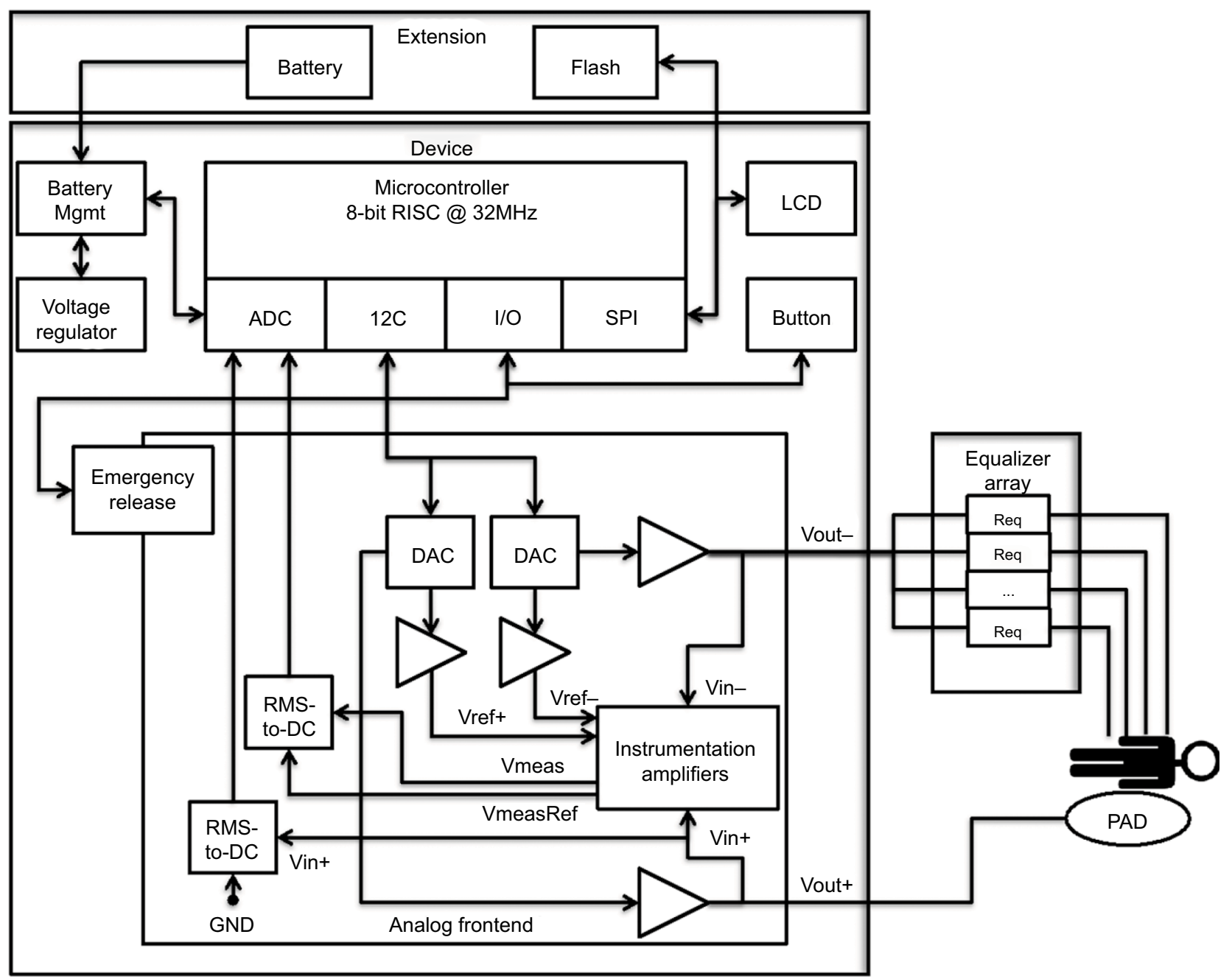

Figure I Block diagram of the medical smart device.

Notes: An analog front end is connected via a digital bus to the microcontroller. The microcontroller adjusts the analog amplifiers to generate the needed current. In order to keep the current at the preselected level, the output voltages $V_{\text {out- }}$ and $V_{\text {out }}$ are continuously measured and adjusted to the changing impedance of the patient. Additionally, the voltage difference between both outputs is measured with a high-precision measurement amplifier network to compensate for surrounding noise (e.g., induced by power-line frequency).

Abbreviations: LCD, liquid-crystal display; ADC, analog to digital converter; DC, direct current; I2C, inter-integrated circuit; I/O, digital input/output; SPI, serial peripheral interface; PAD, electrically conductive self-adhesive pad; DAC, digital to analog converter; RMS, root mean square; GND, ground; RISC, reduced instruction set computer.

manufactured by an original equipment manufacturer. It has been proofed and certified by a notified body of the European Commission (CE 0482).

$$
u(t)=\left\{\begin{array}{c}
0, t \leq 0 \\
U_{\mathrm{ref}} * \beta \frac{t}{T_{\mathrm{Ri}}}, 0<t \leq T_{\mathrm{Ri}} \\
U_{\mathrm{ref}}+U_{\mathrm{ref}} * \alpha^{*} \sin (2 \pi f t), T_{\mathrm{Ri}}<t<\left(T_{\mathrm{max}}-T_{\mathrm{Ro}}\right) \\
U_{\mathrm{ref}} * \beta \frac{T_{\mathrm{max}}-t}{T_{\mathrm{Ro}}},\left(T_{\max }-T_{\mathrm{Ro}}\right) \leq t<T_{\max } \\
0, T_{\max } \leq t \\
\end{array}\right.
$$

$$
\begin{gathered}
\left\{U_{\text {ref }} \mid 0 V \leq U_{\text {ref }} \leq 35 V\right\}\{\alpha \mid 0.01 \leq \alpha \leq 0.4\} \\
\{\beta \mid 0 \leq \beta \leq 0.5\}\left\{T_{\mathrm{Ri}} \mid 10 \mathrm{~s} \leq T_{\mathrm{Ri}} \leq 30 \mathrm{~s}\right\} \\
\left\{T_{\mathrm{Ro}} \mid 10 \mathrm{~s} \leq T_{\mathrm{Ro}} \leq 15 \mathrm{~s}\right\}\left\{T_{\max } \mid 1200 \mathrm{~s} \leq T_{\max } \leq 2400 \mathrm{~s}\right\} \\
\{f \mid 0.01 \mathrm{~Hz} \leq f \leq 1 \mathrm{~Hz}\}
\end{gathered}
$$

A discrete, quantified signal generator algorithm was implemented to create the desired digital waveform patterns, which could then be transformed into continuous analog waveforms by an analog front end. Following the Nyquist-Shannon sampling theorem, the signal generator needs to have a sample rate of at least $0.2 \mathrm{~Hz}$ for an undulation frequency of $0.1 \mathrm{~Hz}$. Analog to digital converters with a resolution of 12 bits are sufficient. With a voltage range of 
0-35 V, the measurement resolution is limited to $8.54 \mathrm{mV}$. With a minimal impedance of $3 \mathrm{k} \Omega$, the minimum current measuring resolution is $2.84 \mu \mathrm{A}$.

For safety reasons, the overall current is continuously checked and limited to a maximum of $540 \mu \mathrm{A}$; in hazardous situations, the complete signal amplification and output of the device are turned off.

The cathodal output is connected to one to nine stainless steel probes via a passive equalizer. The impedance of each inserted needle may vary depending on the applied voltage, needle diameter, location and depth of insertion. Different sets of pre-resistors $(5-50 \mathrm{k} \Omega)$ are used to keep the difference in the output current between needle probes to $<10 \%$.

A stand-alone real-time clock controls the stimulation time. The flash memory stores the log file of treatment-relevant data, such as number of treatments, treatment time and date, electrical parameters and errors, if any. The readout of data can be done comfortably on a PC via Universal Serial Bus.

The user menu is displayed on the liquid-crystal display (LCD). The user is walked through the menu with all information and tests mandatory for medical devices and the selection of the predefined DC stimulation levels. Status information such as remaining treatment time and battery charge condition is also displayed. Errors or hazardous situations are displayed on the LCD and signaled by an alarm tone.

Inflamed or damaged tissue shows pain and is sensitive to pressure (locus dolendi point). In clinical use, the patient indicates the most painful area, which is confirmed by applying pressure (case 1) and if meaningful, in recourse to a magnetic resonance imaging (MRI) examination (cases 2 and 3). Then, the needle is carefully inserted until the patient feels a sharp increase in pain, indicating that the tip of the needle has reached the pathological tissue. Second, the needle is redrawn for a few millimeters, just as to avoid further eliciting pain. Depending on the size of the area to be treated, up to nine needle probes are inserted, usually 1 probe $/ \mathrm{cm}^{2}$. Hereby, the depth of insertion is defined by the location of the area to be treated and ranges from $0.5 \mathrm{~cm}$ (e.g., temporal region, case 1) to $6 \mathrm{~cm}$ (case 2). The conductive skin pad is placed at least $20 \mathrm{~cm}$ away from the needle electrodes. The anodal output is then connected to the skin pad, and the cathodal output to the needle probes via the equalizer. The physician then selects the stimulation level from the menu and starts the stimulation for the next 30 minutes.

\section{Results}

\section{Patient cases}

The following cases refer to single patients, who chose to be treated with the percutaneous DC stimulation on the basis of "compassionate use". These cases do not constitute a clinical study and therefore do not have to be reviewed and approved by an institutional review board (ethics committee). Written informed consent has been obtained by all patients to have the case details and accompanying images published. Importantly, the individual cases presented are not selected super responders; however, they are wholly representative of many other cases we have treated, as we indicate in each of the following three sections.

\section{Cluster headache $(\mathrm{CH})$}

$\mathrm{CH}$ is considered to be the most severe human pain condition, with a prevalence similar to that of multiple sclerosis. Sufferers experience severe, stabbing, one-sided pain in the vicinity of the eye, with a frequency ranging from one to 12 attacks per day. $\mathrm{CH}$ occurs in an episodic form (prevalence $0.1 \%$ ), with attacks mostly during spring and autumn, painfree intervals in between and a chronic form (prevalence $0.01 \%$ ) in which attacks occur for $>11$ months/year. Attacks last between 15 and 90 minutes, and in most cases can only be treated with oxygen inhalation and triptan nasal sprays or injections. Verapamil, topiramate and lithium are commonly used as prophylactic treatments. Available pharmacological treatments are hampered by unwanted side effects, and there is an urgent need for new non-pharmacological treatment alternatives. The annual treatment costs vary between 5,000 and 20,000 euros/patient, resulting in a socioeconomic impact of 1-4 billion euros/year (USA). ${ }^{19-21}$

A 52-year-old male patient had suffered from episodic $\mathrm{CH}$ for 12 years that had turned into a chronic form 1 year before treatment. Daily attacks were treated with oxygen inhalation and a triptan injection. Prophylactic treatment with verapamil had to be stopped 2 years previously after he developed swelling of his legs and bradycardia. Under the aforementioned treatment, his headache diary showed five to eight attacks a day, each lasting 10-30 minutes, with a pain intensity score of $4-10$ on a scale from $0=$ no pain at all to $10=$ unbearable pain. The patient had consulted 12 neurological specialists. He was depressed, sociophobic and suicidal. The desperate patient decided to try a DC stimulation treatment on a compassionate-use basis. He initially received four DC stimulations, one per week, followed by two more DC stimulations at weeks 8 and 12 after the first treatment. DC stimulations lasted 30 minutes each. The anode of the stimulating device was attached to a surface electrode (adhesive pad) on the upper arm. The cathode was attached to a maximum of seven needles $0.3 \times 30 \mathrm{~mm}$. Two needles were placed below the eye beneath the maxilla, and two needles 
were inserted at the temporal region above the temporal branch of the facial nerve and the deep temporal nerve. One needle was placed each at the lateral part of the nose, the lateral end of the eyebrow and at the occipital region above the greater occipital nerve. Depth of insertion varied between 4 and $15 \mathrm{~mm}$, depending on the treated area. A DC of $210 \mu \mathrm{A}$, undulating $\pm 10 \%$ with a frequency of $0.1 \mathrm{~Hz}$, was applied for 30 minutes. After the first treatment, the continuous pain disappeared and the frequency and pain intensity of attacks were reduced by $>50 \%$. The condition improved continuously after each treatment. After the fifth treatment, the frequency of attacks was reduced from five to eight per day to two to four per week, with a pain level of 1-3 and a duration of $<5$ minutes per attack. After the sixth treatment, the attacks had stopped completely (Figure 2 ). At present, $>3$ years later, the patient is still in full remission, completely free of $\mathrm{CHs}$ (all data were taken from the patient's daily headache diary recording attack frequency, pain level and duration of attacks as well as the use of acute medication). In a prospective case series analysis of chronic $\mathrm{CH}$ patients $(\mathrm{n}=18)$ being treated in a similar way, we observed a reduction of at least $50 \%$ in attack frequency, pain intensity, duration of attack and triptan intake in 13 patients 2 weeks after the last percutaneous DC treatment, which lasted for at least 24 weeks (follow-up period).

\section{Acute muscle rupture}

Acute muscle injuries are a major problem for professional and amateur football, soccer and ice hockey players and represent $20-37 \%$ of all time-loss injuries at the men's professional level (18-23\% at the men's amateur level). Available treatment is without proven efficacy and consists of various injections (Actovegin, Traumeel, autologous blood and platelet-rich plasma), physical therapy and extensive rehabilitation to reduce expensive downtime. ${ }^{22}$

A 19-year-old professional soccer player had suffered from an acute muscle rupture of the right rectus femoris during a soccer game. An MRI done the following day showed a 5-6 $\mathrm{cm}$ traumatic lesion of the proximal rectus femoris, accompanied by a massive hemorrhage. The physicians in charge told the patient that he would not be able to play for at least 3 months and referred him to an orthopedist specializing in sports medicine. On the same day, he received the first DC stimulation treatment, followed by four more treatments delivered every second day, each lasting 30 minutes. At each treatment, seven stainless steel needle electrodes, $0.3 \times 30 \mathrm{~mm}$, were inserted around and into the muscle lesion to a depth of 10-50 $\mathrm{mm}$ and connected to the cathode. The anodal skin electrode was positioned at the homolateral waist. A total DC current of $210-540 \mu \mathrm{A}( \pm 10 \%, 0.1 \mathrm{~Hz})$, varying between treatments, was delivered. Other than that the patient received physiotherapy for his lumbar spine only. After the first treatment, the patient felt a noticeable improvement. This was followed by a pain reduction of $80 \%$ after the second treatment and $100 \%$ after the third treatment. After only 10 days, the patient was completely pain free and urged the treating physician to allow him to return to soccer training. However, because the improvement was so unexpectedly fast, a further MRI was scheduled on the eleventh day after



Figure 2 Chronic $\mathrm{CH}$ patient with complete remission to date.

Notes: A male aged 52 years had episodic $\mathrm{CH}$ for 13 years, which was chronic for the past year. The average number of attacks was six per day. The average pain intensity was eight out of 10. He had acute therapy with oxygen, triptan and NSAIDs. The medication use had severe side effects, e.g., tremor, depression, sleep disturbance, inability to work and suicidality. The number of physicians consulted was I0. The number of percutaneous DC treatments was five. Symptoms improved after the first treatment and disappeared within 6 weeks after the last treatment. The patient's $\mathrm{CH}$ remains in complete remission to this day - follow-up $>3$ years. Red bars indicate severity of headache calculated as the product of number, duration and pain intensity of the daily attacks. Blue bars indicate the DC treatments. $x$-axis represents the number of days after the first treatment. Abbreviations: $\mathrm{CH}$, cluster headache; NSAID, nonsteroidal anti-inflammatory drugs; DC, direct current. 
the trauma, which in fact confirmed the regeneration of the muscle (Figure 3). A full return to sports was possible after 3 weeks instead of the expected 3 months. Up to the date of this article, we have treated eight additional cases of acute and chronic muscle fiber rupture with electrical stimulation, all of which showed a comparable clinical recovery.

\section{Ankle sprain with rupture of the anterior talofibular ligament}

The ankle is the most commonly injured area of the body. Approximately $25 \%$ of all injuries of the musculoskeletal system involve inversion injuries of the ankle, and $\sim 50 \%$ of these injuries are sports related (soccer, basketball, running, dancing). The anterior talofibular ligament is damaged in twothirds of all injuries, ranging from simple sprain to complete rupture. Treatment is based upon the stages of biological ligament healing - the proliferation phase from 6 weeks to 3 months after the trauma, and the remodeling phase up to 1 year after trauma - and consists of rest, ice, immobilization, support and functional treatment. ${ }^{23}$

A 25-year-old female suffered from a severe traumatic injury of the right ankle during a soccer game. The ankle was swollen and painful. The MRI showed a rupture of the anterior talofibular ligament. On days 1, 4 and 10 following the trauma, the patient received 30 minutes of DC EF stimulation. Six stainless steel needle electrodes, $0.3 \times 30 \mathrm{~mm}$, were inserted at the lateral aspect of the ankle, close to and directly into the most painful area, which corresponded to the lesion of the ligament. The depth of insertion was 5-10 mm, depending on the location of the needle. All needles were connected to the cathode. The anodal skin electrode was positioned at the homolateral thigh. A total DC current of $270 \mu \mathrm{A}( \pm 10 \%$, $0.1 \mathrm{~Hz}$ ) was delivered. The day after the first treatment (day 2 post-trauma), pain and swelling were significantly reduced and the patient began walking without crutches. On day 4 , before the second treatment, walking was almost normal. On the day of the third treatment (day 10 after trauma), she was asked to start soccer training again. To verify the unexpected clinical progress, a second MRI was undertaken on day 14 after trauma, which showed a significant regeneration of the ruptured ligament. A third MRI, 6 weeks after the injury, showed an almost normal ligament, without the expected typical signs of secondary scar tissue (Figure 4). A total of 10 other patients with ankle sprain demonstrated a likewise clinical uncommon reduction in swelling and pain within up to three treatments.

We include two videos (Videos S1 and S2) as supplementary materials (link: https://www.boewing-molsberger. de/publications supplementary info/).

The first shows a patient who had suffered from severe $\mathrm{CH}$ for 6 years and after three percutaneous DC stimulations, reports a marked improvement. The video also shows the treatment procedure and a follow-up over 10 weeks. The second shows a patient with repeated muscle ruptures and pain, which severely limited him from playing professional ice hockey. After four percutaneous DC stimulation
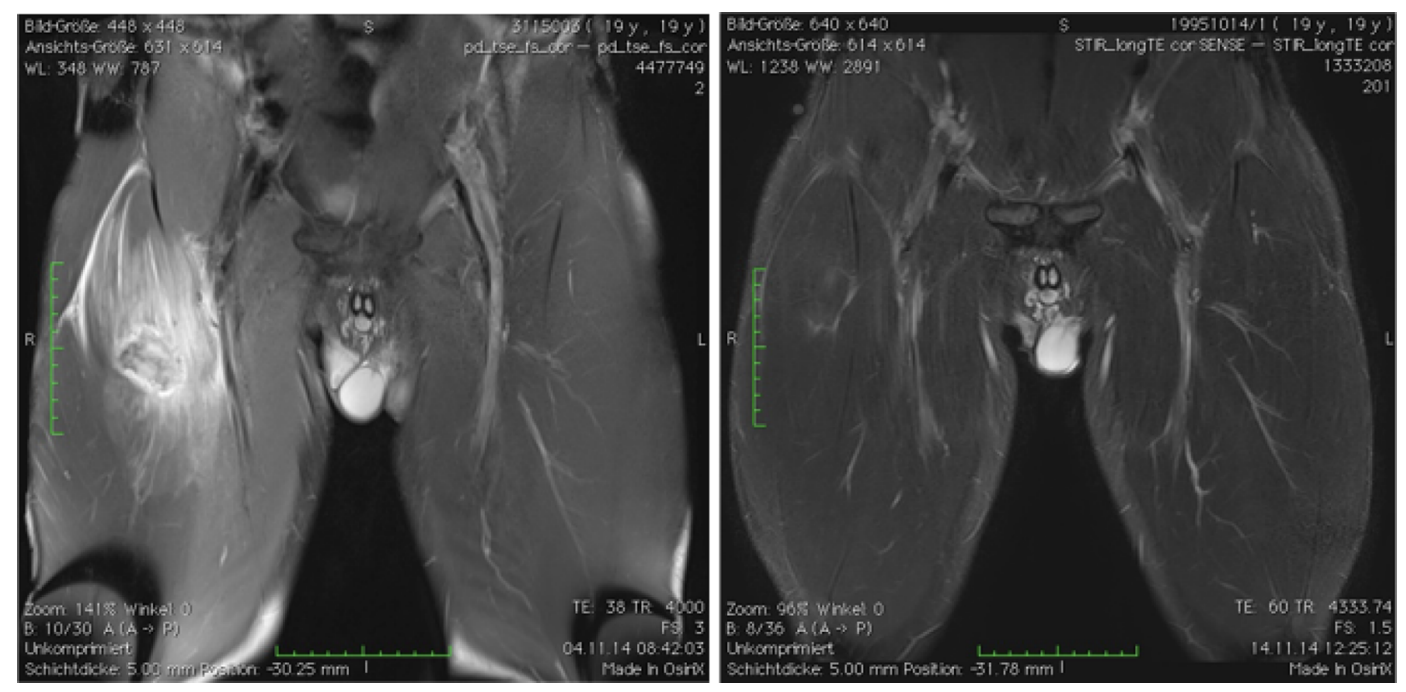

Figure 3 Acute muscle rupture of the rectus femoris.

Notes: A 19-year-old professional soccer player suffered from acute muscle rupture of the thigh during the second half of the game. He was taken out of sports for an expected 3 months minimum. Five percutaneous DC treatments were carried out over a 2-week period. He had noticeable improvement after the second treatment, was pain free after the fifth treatment and resumed sport after 3 weeks. Left: MRI on the day of the injury - hematoma with ruptured muscle fibers. Right: MRI after five DC treatments - II days after trauma.

Abbreviations: MRI, magnetic resonance imaging; DC, direct current. 


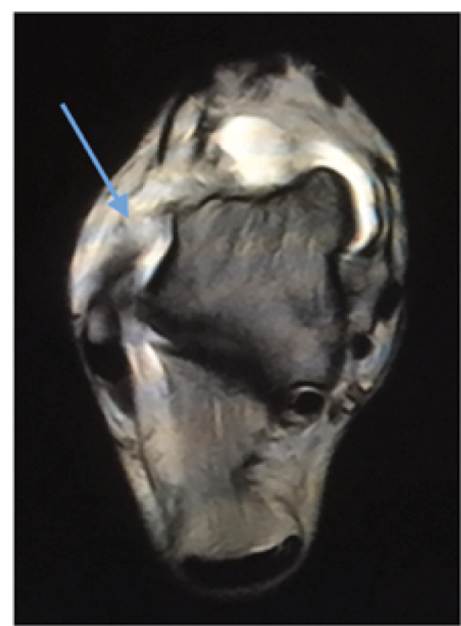

Day 2 after trauma

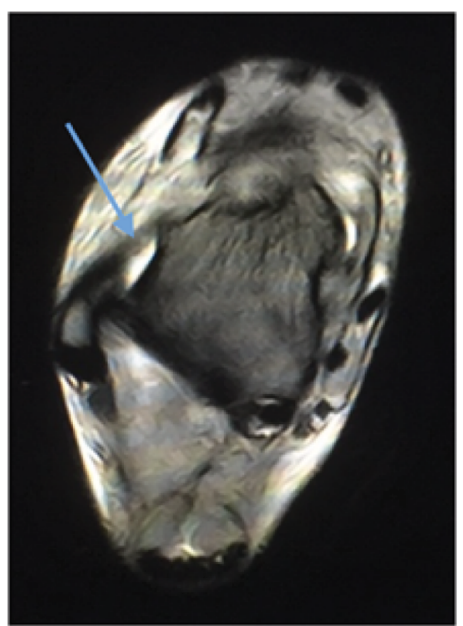

Day 14 after trauma

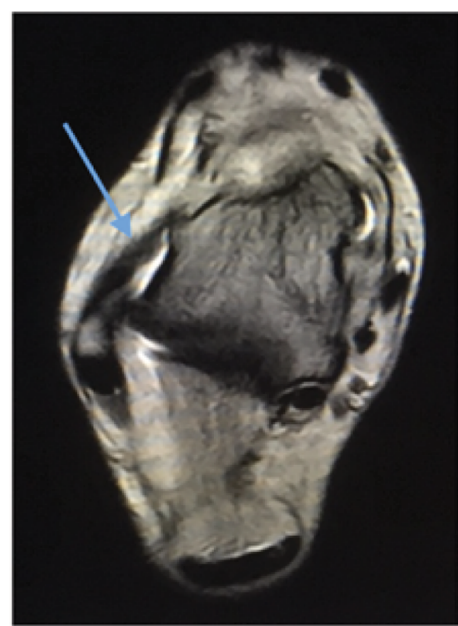

6 weeks after trauma

Figure 4 Ankle sprain with rupture of the anterior talofibular ligament.

Notes: A 25-year-old female soccer player suffered from a severe traumatic injury of the ankle during a soccer game. The ankle was severely swollen and painful. The MRI showed a rupture of the anterior talofibular ligament. Percutaneous DC treatments were applied on days I, 4 and I0 after trauma. Immediately after the first treatment, pain and swelling were significantly reduced and she started to walk without crutches. On day 4, before the second treatment, walking was almost normal. On the day of the third treatment (day 10 after trauma), she was asked to start soccer training again. To verify the unexpected clinical progress, a second MRI was undertaken on day I4 after trauma, showing significant regeneration of the ruptured ligament. The third MRI, 6 weeks after trauma, showed an almost completely new ligament, without the expected typical signs of secondary scar tissue. The arrows indicate the anatomical structure of the anterior talofibular ligament.

Abbreviation: MRI, magnetic resonance imaging.

treatments, he has been able to play 20 games without pain or limitation. We deliberately chose different patients from the ones detailed in the paper to show that these treatments are effective for most of our patients.

\section{Discussion}

The preceding cases - a severe chronic nerve problem, an acute traumatic muscle injury and an acute joint sprain with an injured ligament - are different in nature, involving different tissues and conditions that heal over hugely variable time periods. However, after DC EF stimulation, they all followed a similar pattern of improvement. First, a significant reduction in inflammatory signs such as pain, swelling and redness was observed within 1-3 hours after treatment. This first effect lasted up to several days. Second, after two to six treatments, the anti-inflammatory effect stabilized and further improvement up to a complete remission or recovery followed. These diverse conditions normally are difficult to treat and resolve only slowly, if at all. Using the same electrical stimulation technique, however, induced 1) strong early pain reduction; 2) anti-inflammatory effects and 3 ) long-term improvements that were strikingly more effective than current clinical treatments. Here, we present two hypotheses to explain these unusual and compelling clinical observations.

\section{Fast pain reduction and anti-inflammatory effect}

It is known that local acidosis and an increased concentration of proinflammatory cytokines is associated with local pain, inflammation and swelling. For example, Shah et $\mathrm{al}^{7}$ measured the local acidotic state and various proinflammatory cytokines such as CGRP, bradykinin, SP, TNF-alpha and IL-1beta in active muscle trigger points. It is known further that a DC current induces an ionic current in biological tissue, with cations moving toward the cathode (negatively charged electrode) and anions moving toward the anode (positively charged electrode). The DC treatment device delivers the current at the cathodal site via metal probes inserted directly into the inflamed tissue. A transdermal connective skin pad is used at the anode site. At the cathode, electrolysis occurs according to the formula $2 \mathrm{H}_{2} \mathrm{O}+2 \mathrm{e}^{-} \rightarrow \mathrm{H}_{2}+2 \mathrm{OH}^{-}$. The liberated $\mathrm{OH}^{-}$ions lead to a locally more basic $\mathrm{pH}$ value. This shift in the $\mathrm{pH}$ value from acidosis to alkalosis spreads into the surrounding tissue by diffusion during the 30-minute treatment time, thus buffering the acidotic state and reducing pain and inflammation. Although this mechanism has not been observed directly in the ruptured muscle, it is a regular observation in tissue culture where local $\mathrm{pH}$ changes arise after long periods around the electrodes and have been observed directly in ex vivo brain slice preparations where 
a pulsed EF was used to overcome the $\mathrm{pH}$ and acidosis changes. ${ }^{24}$ Furthermore, the electrically charged proinflammatory cytokines will react to the induced EF through electrostatic forces and electrophoresis, perhaps concentrating some and reducing the concentration of others in specific locations. Although requiring direct laboratory testing in the live muscle, we suggest that the buffering of local acidosis and the influence on cytokines are likely to explain the fast decongestant and pain-reducing effect.

\section{Long-term improvement}

Static electric DC fields guide and stimulate the process of inflammation and regeneration of wounded tissue. The wound relative to the intact tissue always represents the cathodal side of the current. Non-mammalian vertebrates like the urodele or the axolotl that can regenerate complete amputated limbs and full organs (heart, brain, eye lens) exhibit high EFs up to $400 \mathrm{mV} / \mathrm{mm}$ at the injured site. ${ }^{10}$ Mammalian vertebrates have lost this full regenerative capacity and only exhibit wound-induced DC currents from 10-30 $\mu \mathrm{A} / \mathrm{cm}^{2}$ (human fingertips) to $300 \mu \mathrm{A} / \mathrm{cm}^{2}$ (guinea pigs) and an $\mathrm{EF}$ of up to $140 \mathrm{mV} / \mathrm{mm} .{ }^{8}$ Thus, it can be argued that the intensity of the current and the strength of the resulting EF are crucial for the speed and the completeness of the regeneration. It is thought that an increased current alone, over only a short time span, will suffice to trigger long-lasting regenerative processes. ${ }^{25}$ The DC stimulation therapy described earlier delivers a cathodal electric current of 30-540 $\mu \mathrm{A} /$ needle to the injured site. This results in a 10-20-fold reinforcement of the naturally occurring wound DC, one to two times a week, for 30 minutes each time. It is hypothesized that the unexpectedly fast and high-quality regeneration of muscles and ligaments observed is in fact triggered by the therapeutically increased DC at the injured tissue.

In closing, it is important to comment on the remarkable ability of a single intervention, a DC EF to cure a series of pathologies with a widely varying etiology. The body generates its own DC electrical signals, and there is unequivocal evidence that they play several roles in orchestrating the complexity and variability of the different types of cellular responses that collectively are required for successful wound healing. ${ }^{6,813} \mathrm{DC}$ electrical stimulation regulates cell proliferation, migration and differentiation of many cell types in cell-specific ways. This includes nerve, muscle, epithelial and endothelial cells; macrophages; fibroblasts; and mesenchymal stem cells, among many others. Not only do these cell types behave in cell-specific ways when stimulated, they also release multiple cell-specific growth factors and neurotransmitters and regulate the expression of growth factor, cytokine and neurotransmitter receptors. For example, DC stimulation around blood vessels stimulates new vessel sprouting, directed endothelial cell migration and the release of vascular endothelial growth factor (VEGF) ${ }^{6,8}$ effects, which may underpin the improvements seen in $\mathrm{CH}$. Because of this repertoire of cell-specific responses to a DC EF, we should expect different wounds/pathologies to react to DC stimulation by selecting the appropriate combination of cellular behaviors and responses that will enhance this specific healing situation.

There is nothing "magical" about a unitary intervention improving multiple different pathologies. By contrast, it reflects and emphasizes the relative ubiquity of woundinduced electrical signals, their multiple and specific cellular and molecular targets and consequently, the multiplicity of ways in which they may be beneficial clinically.

\section{Conclusion}

TEPs generate small EFs that orchestrate cell differentiation, division and migration in many wound-healing and tissueregenerating processes. Here, we presented a new kind of bioelectric stimulation device delivering small direct currents and consequent EFs mimicking the TEP-induced EFs. Three cases of different etiology illustrate the potentially powerful clinical impact of this promising electroceutical treatment approach: $\mathrm{CH}$, a very severe and costly pain condition; muscle rupture, a frequent sports problem for which treatment of proven effectiveness is still lacking; and ankle sprain, the most frequent trauma injury for humans. Each condition showed a more rapid and lasting improvement that is not observed with conventional treatments. As indicated, we have many more cases showing the same pattern of pain reduction and lasting regeneration in soft-tissue conditions that would have otherwise healed incompletely and/or more slowly. We are convinced therefore that this bioelectric DC stimulation regime represents a transformative new approach for the regenerative treatment of soft-tissue conditions. Randomized controlled trials are scheduled for the near future, as well as laboratory work aimed at reverse engineering on a cellular level of the clinically observed regenerative effect.

\section{Acknowledgments}

We are grateful to the orthopedic MDs Lutz Neumann (case 2) and Andreas Graeb (case 3) for their contributions. We also thank Wolfgang Schumann for the critical reading of the manuscript and Harald Fischer for the technical description of the device. 


\section{Disclosure}

Albrecht Molsberger is the CEO of the company, which builds the percutaneous DC stimulator ( $\mathrm{CHP} \mathrm{GmbH})$. He holds one granted and several pending patents on the procedure. He has been receiving funding from the German government for the technical and clinical development of the treatment. Colin D McCaig has no conflicts of interest in this work.

\section{References}

1. Nnoaham KE, Kumbang J. Transcutaneous electrical nerve stimulation (TENS) for chronic pain. Cochrane Database Syst Rev. 2008;(3): CD003222.

2. Shamji MF, De Vos C, Sharan A. The advancing role of neuromodulation for the management of chronic treatment-refractory pain. Neurosurgery. 2017;80(3):S108-S113.

3. Petersen EA, Slavin KV. Peripheral nerve/field stimulation for chronic pain. Neurosurg Clin N Am. 2014;25(4):789-797.

4. Zeng Z, Yan M, Dai Y, Qiu W, Deng S, Gu X. Percutaneous bipolar radiofrequency thermocoagulation for the treatment of lumbar disc herniation. J Clin Neurosci. 2016;30:39-43.

5. García Naranjo J, Barroso Rosa S, Loro Ferrer JF, Limiñana Cañal JM, Suarez Hernández E. A novel approach in the treatment of acute whiplash syndrome: ultrasound-guided needle percutaneous electrolysis. A randomized controlled trial. Orthop Traumatol Surg Res. 2017;103(8):1229-1234.

6. McCaig CD, Song B, Rajnicek AM. Electrical dimensions in cell science. J Cell Sci. 2009;122(23):4267-4276.

7. Shah JP, Danoff JV, Desai MJ, et al. Biochemicals associated with pain and inflammation are elevated in sites near to and remote from active myofascial trigger points. Arch Phys Med Rehabil. 2008;89(1):16-23.

8. Mccaig CD, Rajnicek AM, Song B, Zhao M. Controlling cell behavior electrically: current views and future potential. Physiol Rev. 2005;85(3):943-978.

9. Rouabhia M, Park H, Meng S, Derbali H, Zhang Z. Electrical stimulation promotes wound healing by enhancing dermal fibroblast activity and promoting myofibroblast transdifferentiation. PLoS One. 2013;8(8):e71660.

10. Borgens RB, Vanable JW, Jaffe LF. Bioelectricity and regeneration. I. Initiation of frog limb regeneration by minute currents. $J$ Exp Zool. 1977;200(3):403-416.
11. Leppik LP, Froemel D, Slavici A, et al. Effects of electrical stimulation on rat limb regeneration, a new look at an old model. Sci Rep. 2015;5:18353.

12. Baer ML, Colello RJ. Endogenous bioelectric fields: a putative regulator of wound repair and regeneration in the central nervous system. Neural Regen Res. 2016;11(6):861-864.

13. Zhao M, Song B, Pu J, et al. Electrical signals control wound healing through phosphatidylinositol-3-OH kinase-gamma and PTEN. Nature. 2006;442(7101):457-460.

14. Borgens RB, Jaffe LF, Cohen MJ. Large and persistent electrical currents enter the transected lamprey spinal cord. Proc Natl Acad Sci U S A. 1980;77(2):1209-1213.

15. Baer ML, Henderson SC, Colello RJ. Elucidating the role of injuryinduced electric fields (EFs) in regulating the astrocytic response to injury in the mammalian central nervous system. PLoS One. 2015;10(11):e0142740.

16. Zhao Z, Watt C, Karystinou A, et al. Directed migration of human bone marrow mesenchymal stem cells in a physiological direct current electric field. Eur Cell Mater. 2011;22:344-358.

17. Hoare JI, Rajnicek AM, Mccaig CD, Barker RN, Wilson HM. Electric fields are novel determinants of human macrophage functions. J Leukoc Biol. 2016;99(6):1141-1151.

18. Balint R, Cassidy NJ, Cartmell SH. Electrical stimulation: a novel tool for tissue engineering. Tissue Eng Part B Rev. 2013;19(1):48-57.

19. May A, Leone M, Áfra J, et al; EFNS Task Force. EFNS guidelines on the treatment of cluster headache and other trigeminal-autonomic cephalalgias. Eur J Neurol. 2006;13(10):1066-1077.

20. Gaul C, Finken J, Biermann J, et al. Treatment costs and indirect costs of cluster headache: a health economics analysis. Cephalalgia. 2011;31(16):1664-1672.

21. Rozen TD, Fishman RS. Cluster headache in the United States of America : demographics, clinical characteristics, triggers, suicidality and personal burden. Headache. 2012;52(1):99-113.

22. Linklater JM, Hamilton B, Carmichael J, Orchard J, Wood DG. Hamstring injuries: anatomy, imaging, and intervention. Semin Musculoskelet Radiol. 2010;14(2):131-161.

23. van den Bekerom MPJ, Kerkhoffs GMMJ, McCollum GA, Calder JDF, van Dijk CN. Management of acute lateral ankle ligament injury in the athlete. Knee Surg Sport Traumatol Arthrosc. 2013;21(6):1390-1395.

24. Feng JF, Liu J, Zhang L, et al. Electrical guidance of human stem cells in the rat brain. Stem Cell Reports. 2017;9(1):177-189.

25. Reid B, Zhao M. The electrical response to injury: molecular mechanisms and wound healing. Adv Wound Care. 2014;3(2):184-201. 


\section{Supplementary materials}

Video SI https://vimeo.com/261400362.

Video S2 https://vimeo.com/2614067/0.

Medical Devices: Evidence and Research is an international, peerreviewed, open access journal that focuses on the evidence, technology, research, and expert opinion supporting the use and application of medical devices in the diagnosis, monitoring, treatment and management of clinical conditions and physiological processes. The identification of novel devices and optimal use of existing devices which will lead to improved clinical outcomes and more effective patient management and safety is a key feature. The manuscript management system is completely online and includes a quick and fair peer-review system. Visit http://www. dovepress.com/testimonials.php to read real quotes from authors.

Submit your manuscript here: https://www.dovepress.com/medical-devices-evidence-and-research-journal 